\title{
A DATA FUSION APPROACH TO AUTOMATED VEHICLE DETECTOR TESTING
}

\author{
C. Arthur MacCarley \\ California Polytechnic State University, San Luis Obispo, California, USA \\ Neils Nesse \\ Loragen Corporation, San Luis Obispo, California, USA \\ John Slonaker \\ California Department of Transportation, Division of Research and Innovation
}

\begin{abstract}
Vehicle presence detectors have become critical elements of traffic management systems, including applications ranging from intersection signal control to freeway congestion monitoring. The need to assess the accuracy and attributes of each of the many types of sensors motivated the California Department of Transportation to construct the Traffic Detector Testbed on I-405 in Southern California. With up to ten detectors of different types under concurrent test in each of six lanes, a means for automating the testing process became imperative, since traditional humanverification methods were not practical. This paper describes the design and implementation of an automated data acquisition and verification system that processes data from all detectors along with that of a reference image processing system, to create a composite ground truth record against which individual detector performance is assessed. The system architecture, data fusion methodology, computer vision methods, operator interface and system performance results are discussed.
\end{abstract}

Index Terms - Video traffic detection, automated data reduction, detector verification, traffic monitoring, data fusion, ITS.

\section{INTRODUCTION}

The detection of the presence, speed and/or length of vehicles on roadways is increasingly recognized as critical for effective roadway congestion management and safety. The use of vehicle presence sensors is common practice for traffic volume measurement, and control of signalized intersections and ramp meters. In addition, vehicle speed and classification from length are important for automated incident detection and the characterization and prediction of traffic demand. Real-time detection is also used for actuation of automated driver information systems, for example, the Caltrans Automated Warning System (CAWS) on I-5 in central California [1].

Among the sensing mechanisms used for vehicle detection are changes in inductance, changes in magnetic field strength, video image processing, microwave RADAR, optical transmission, laser transmission or pulse time-of-flight, and acoustic signature discrimination [2,3]. Detectors based on each method are known to have advantages and limitations which make them appropriate for some applications, but inappropriate for others. The need to assess the relative accuracy and attributes of each type of sensor against a set of common, objective standards motivated the California Department of Transportation to construct a detector/sensor testbed on Interstate 405 in Southern California, one of the highest-volume freeways in the state.

The challenge in any large-scale roadway test involving individual vehicle records is the creation of a "ground truth" reference dataset that represents the actual microscopic history of the traffic. This traditionally requires human verification of each vehicle, either by direct observation or from playback of video tapes. A novel feature of the Caltrans testbed is an automated data acquisition and verification process, which reduces the workload of verifying individual detection events by combining the results of all detectors under test and pre-screening unambiguous cases for which a consensus is obvious. Manual verification by a human operator is required only for reported detections for which there is not clear agreement, in a weighted sense, between all detectors including the video detection function of the verification system itself. A digital image is acquired by the system for every reported detection, and a set of graphical computer tools are provided to facilitate rapid manual verification of ambiguous detections by reference to this image. Individual performance statistics are then automatically generated by comparison of the results produced by each detector with the confirmed ground truth dataset. Without such a labor-saving system, $100 \%$ data verification would not be practical, considering the large traffic volume (typically 6000 vehicles per hour) and as many as ten detectors tested in parallel. We focus here on the automated data reduction and verification process, and the methods used by the video processor for robust detection and estimation of the speed of vehicles.

\section{SYSTEM ARCHITECTURE AND FEATURES}

The Video Vehicle Detector Verification System (V2DVS) deployed in the Caltrans Detector Testbed is physically comprised of a cluster of rack-mount Linux computers (field machines), one per lane, and a LAN or Internet-connected central database server. In addition, a PC-based client program facilitates remote monitoring and control of all field machines, and manual ground truth verification. Each field machine interfaces a video camera positioned on an overcrossing above an assigned traffic lane. Detectors under test are connected to the V2DVS, and each reported vehicle detection generates a record consisting of a JPEG compressed image and the time of arrival and speed of the vehicle. A maximum of eight contact closures or logic level inputs, and an unlimited number of network or serial signal inputs are accepted for each lane. Although only one test site is currently operational, multiple test sites are supported. 


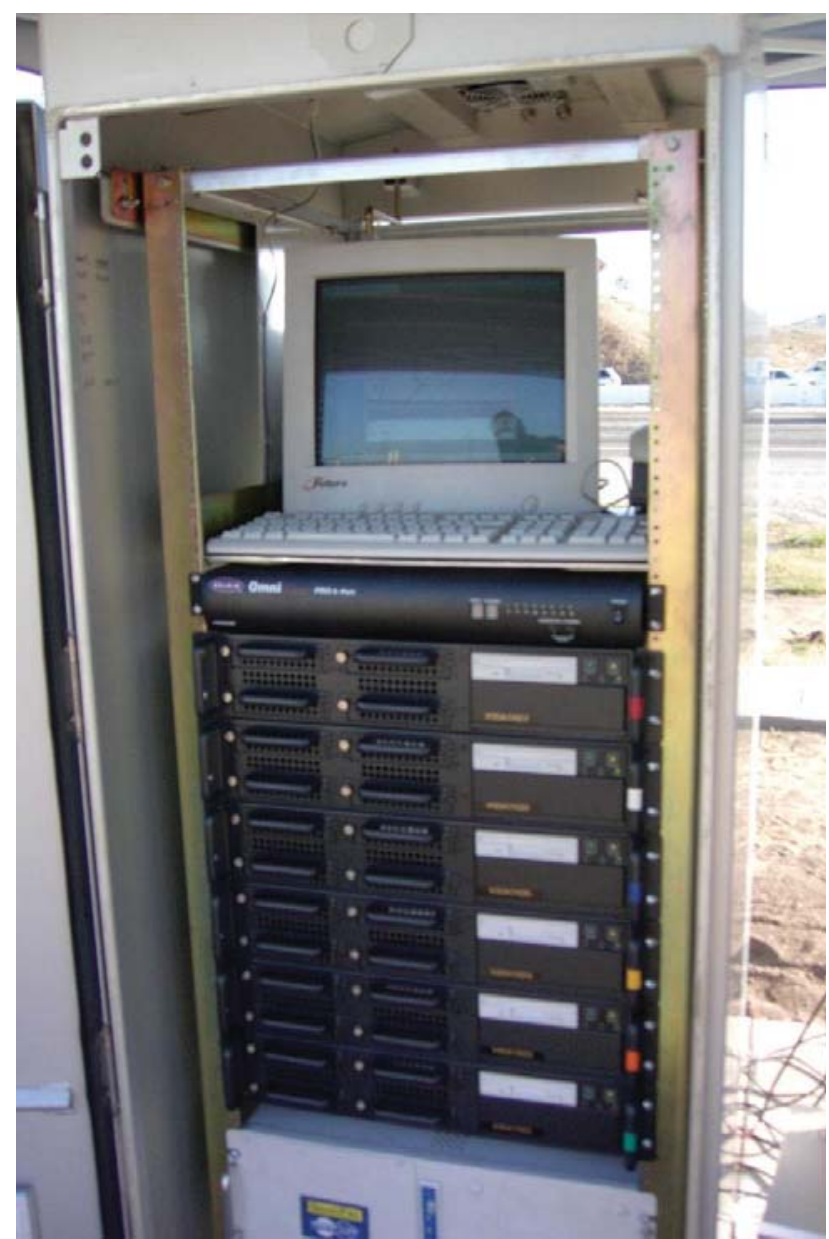

Figure 1. V2DVS field computers in Type 334 cabinet.

At maximum traffic capacity, as many as 96,000 records per hour per site may be generated per site. The primary test site is equipped with poles on the median and roadside on which widearea detectors are mounted. Each of the traffic lanes has duplex inductive loops. The camera structures on the overcrossing provide mounting points for other over-lane detectors. Detector electronics and the V2DVS field machines are housed in three roadside Caltrans Type 334C cabinets, one shown in Figure 1.

Video verification uses down-looking high-resolution NTSC video cameras placed 10 meters above each traffic lane as shown in Figure 2. Cameras have manual electronic shutters set at 1/4000 sec. to prevent image blur from moving vehicles. The field of view of each camera extends from immediately below the overcrossing deck to approximately 25 meters down-road, to contain most of the zones of detection used by different detectors; exceptions are video-based detection systems which use far-field approaching or departing traffic views. This camera deployment is optimal for testbed research purposes, but is not considered practical for general-purpose traffic monitoring since individuallane overhead camera placements are usually only possible on overcrossings.

Each detector under test signals the system for each vehicle detected. Signaling may be real-time in which a record is

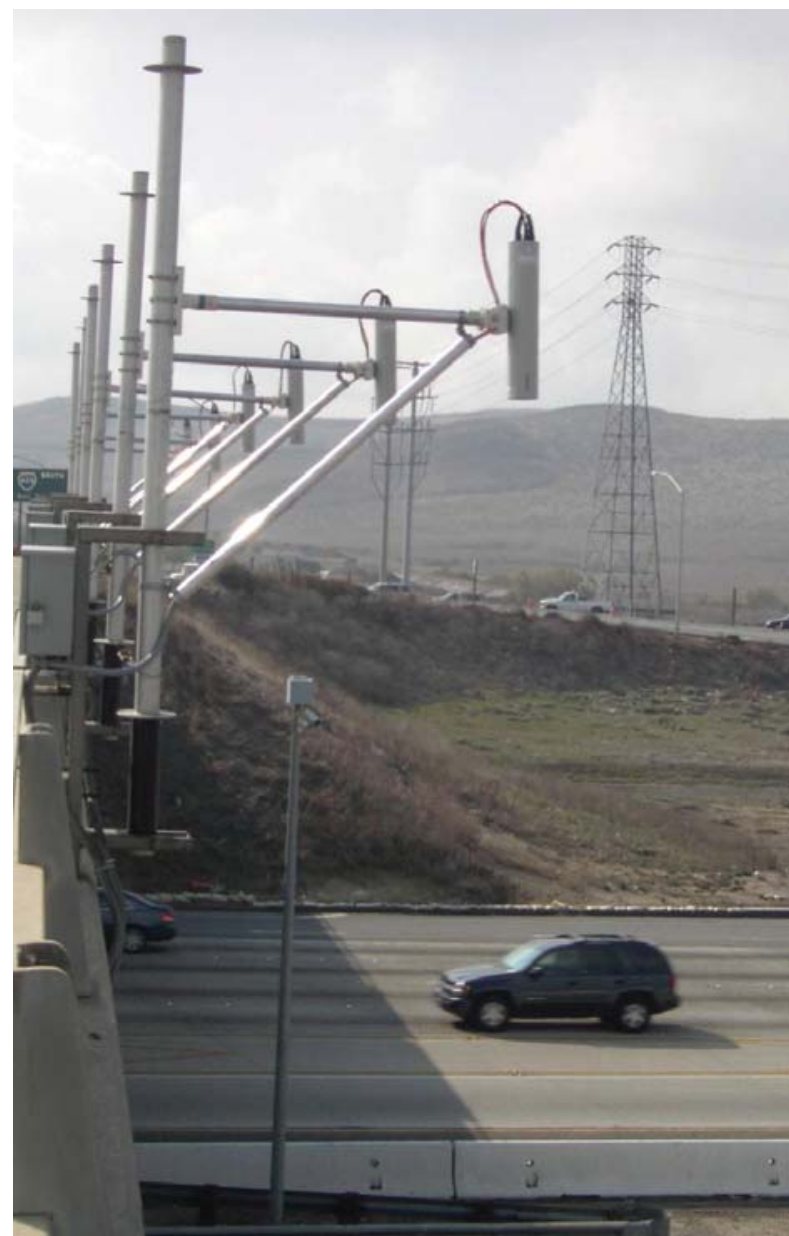

Figure 2. Down-looking video cameras above each lane.

acquired immediately, or delayed up to 5 seconds after the actual time of detection to accommodate some detectors that have processing delays. The image acquisition function maintains a circular buffer of the most recent 300 video images (fields) acquired at 60 video fields per second for this purpose.

Signaling is accepted by two physical methods: 1 . hardware signaling (contact closure or open-collector active-low logic level), and 2. network or serial port signaling. Most detectors use hardware signaling. The latter method provides a means for a detector to report the exact time of detection for delayed signaling, and additional information that may be included in the event record. A typical data string, if displayed on a terminal, might be:

\section{A $50601011430155065.0150 .0 \mathrm{~S}$}

Where:

Detector code $=\mathrm{A}$ (each detector is assigned single letter ID)

Lane $=5$

Year $=2006$, Month $=$ January, Day $=1$

Time $=2: 30 \mathrm{PM}$ and 15.50 seconds

Speed $=65.0 \mathrm{MPH}$

Distance offset from baseline (if not constant $)=150.0$ feet

Site Code $=$ S (Sand Canyon) 
For real-time signaling, the most recently acquired image is recorded along with the exact time that the signal was received, accurate to 0.001 second. For delay-time signaling, the queued image closest to the time of detection is stored for that record. To assure exact time synchronization for delayed detectors which report specific detection times, V2DVS provides an NTP (Network Time Protocol) local time server which must be referenced by any detector which uses delay-time signaling. Different detection zones and processing delays are accommodated for each detector, to be discussed later. All records (image and data) are stored locally on each field machine, and automatically pushed via SFTP on a bandwidth-available basis to the central server, which stores data objects in a MySQL [4] database, and images in indexed directories. Local storage capacity allows tests up to twenty days in duration prior to the need to off-load data to the server. While connectivity between elements at the field site(s) is provided by a $100 \mathrm{Mbps}$ LAN, the field site(s) are connected to the server via multiple networks including a relatively slow $802.11 \mathrm{~b}$ wireless link. As a result, up to 24 hours may be required to transfer a few hours of test data and images. Alternatively, the server can be set up on-site to avoid these bandwidth limitations.

Computer control of the camera iris is critical for maintaining an optimal and consistent scene intensity level for image processing. Each field machine provides this function via a $68 \mathrm{HC} 12$ microcontroller interface and lens motor driver subsystem. The field machines may be controlled locally or remotely from any Internet-connected computer via the V2DVS client application, including manual override of the camera iris, focus and zoom controls. The client remote control screen is shown in Figure 3.

\section{REFERENCE VEHICLE DETECTION AND VEHICLE SPEED CALCULATION}

As previously mentioned, the field machine for each lane acquires a record including an image for each signaled detection event. But they also acquire their own detection record by processing the video stream and acquiring an image when each vehicle arrives at a baseline position in the field of view of the camera. This serves as a reference image and provides vehicle speed data needed for proper correlation between detectors with different detection zones. A typical detection verification image is shown in Figure 4.

As with any video-based traffic detector, the V2DVS is susceptible to errors due to the inherent limitations of natural scene illumination at various sun angles or from vehicle headlights. A major problem is false detection due to shadows, especially those cast by vehicles in adjacent lanes. These image artifacts often meet the same texture, contrast and motion requirements as an actual vehicle, and can be therefore be incorrectly detected. Long shadows are a particular issue during morning and evening rush hours, which are the most interesting traffic conditions for detector testing. A special effort was made to improve the ability to distinguish shadows from actual vehicles, and to also correctly detect vehicles imbedded in or connected to shadows cast by other vehicles.

The most successful approach involves tests of texture and contrast, such as those used by $[5,6]$, but primarily relies on the continuity of a shadow originating from an adjacent lane.

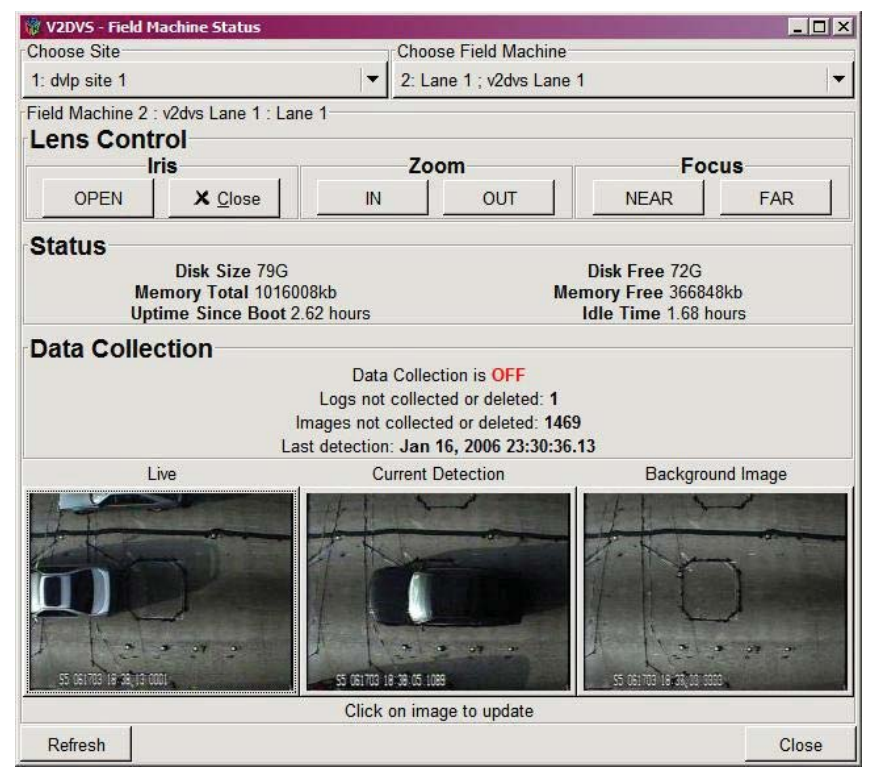

Figure 3. Remote control window of client application.

Processed image areas include adjacent lanes on either side of the lane under test. A long-shadow situation is illustrated in Figure 6, which shows the video processor user interface in diagnostic mode with optical flow scan lines visible. In this example, the continuity of the vehicle shadow extending from the adjacent lane into the detection zone effectively discriminates the shadow from an actual vehicle, while texture tests avoid incorrect rejection of a possible vehicle embedded in the shadow.

The ability to compare the results of several detectors, each that identify the same vehicle at different positions on the roadway, requires knowledge of the vehicle speed. Since few detectors under test report vehicle speeds, the V2DVS must generate an accurate measurement of speed for each vehicle detected. A multitiered algorithm is used, shown in a high-level view in Figure 5. The position of the vehicle leading edge is tracked as it passes through the camera field of view. Position samples are stored for each video field, vetted for forward progression and reasonableness, and then used to define a linear equation by leastsquares curve fitting. The linear coefficient is the estimated speed value. The sample variance about the linear function is tested, and if found to be excessive, the algorithm reverts to one of two alternative methods: A histogram is generated for speed calculated from the elapsed times between every reasonable pairing of position samples. The median speed value from the histogram serves as the vehicle speed estimate. Or in very sparse data situations, detection may be based on simple volume intensity change in two consecutive zones along the path the vehicle, and speed calculated as time-of-flight between the zones (a method commonly used by video-based intersection vehicle detectors).

A valid speed measurement is required as a qualification for a valid vehicle detection. Video detection accuracy is affected primarily by the scene illumination, and secondarily by the characteristics or lane position of the vehicle. Manually confirmed accuracy over the range of possible daylight conditions is reported in Table 1. 


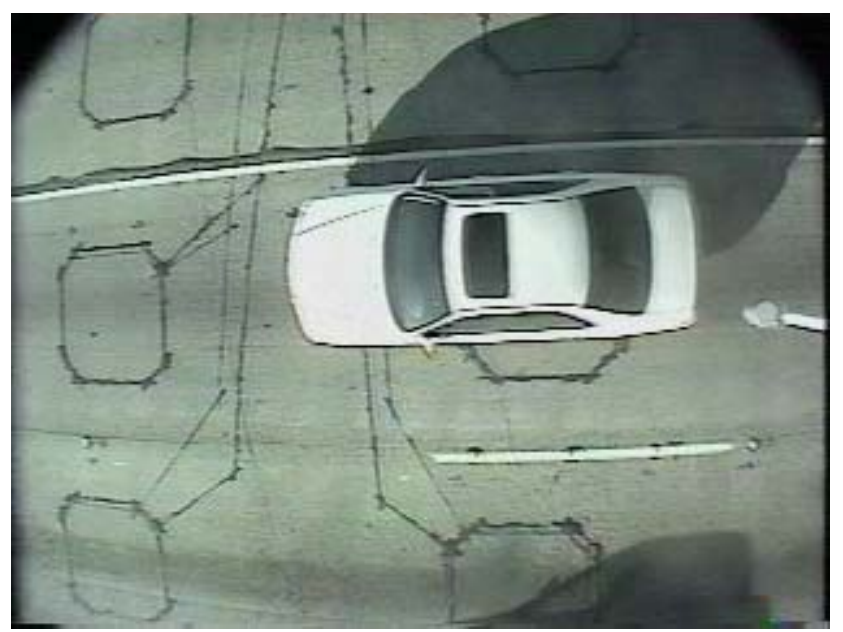

Figure 4. Typical detection image.

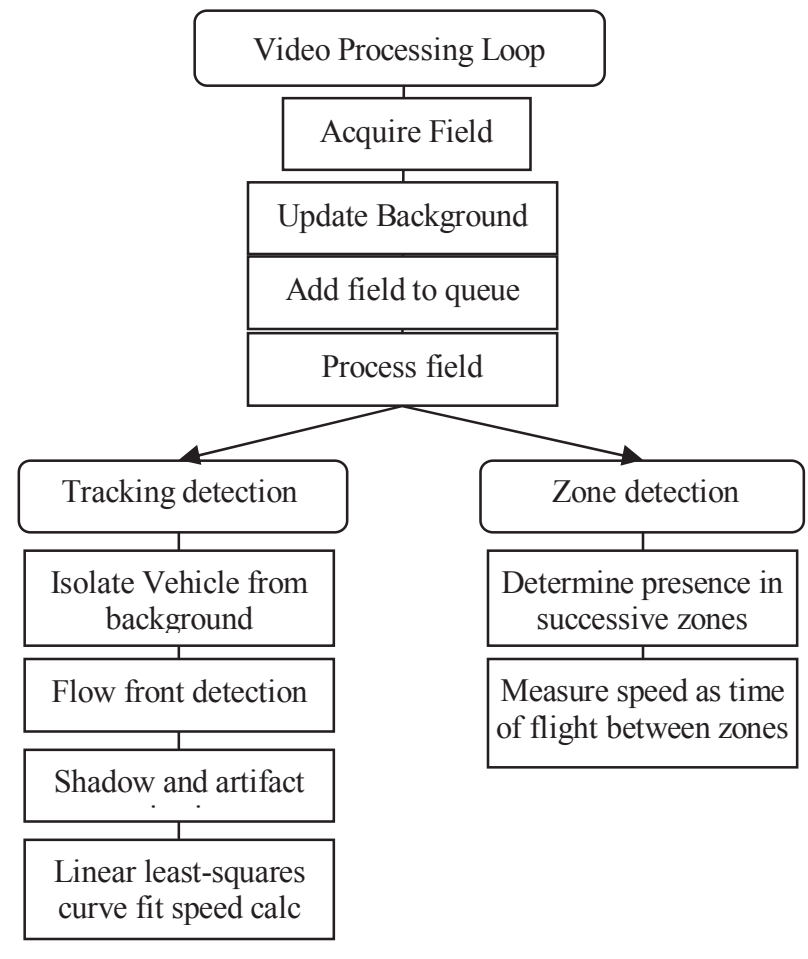

Figure 5. Video vehicle detection and speed measurement.

\section{AUTOMATED DATA VERIFICATION}

In post-processing, a composite ground truth dataset is derived from all detector data, based upon user-selectable confidence criteria. The primary automation task is to identify records of the same vehicle reported by different detectors having different detection zones and processing delays. Adjusted detection times are calculated from the distance (offset) of each zone from a baseline position, using the assumption of constant vehicle speed over the offset interval. As previously discussed, speed is estimated by video processing for each proximate candidate

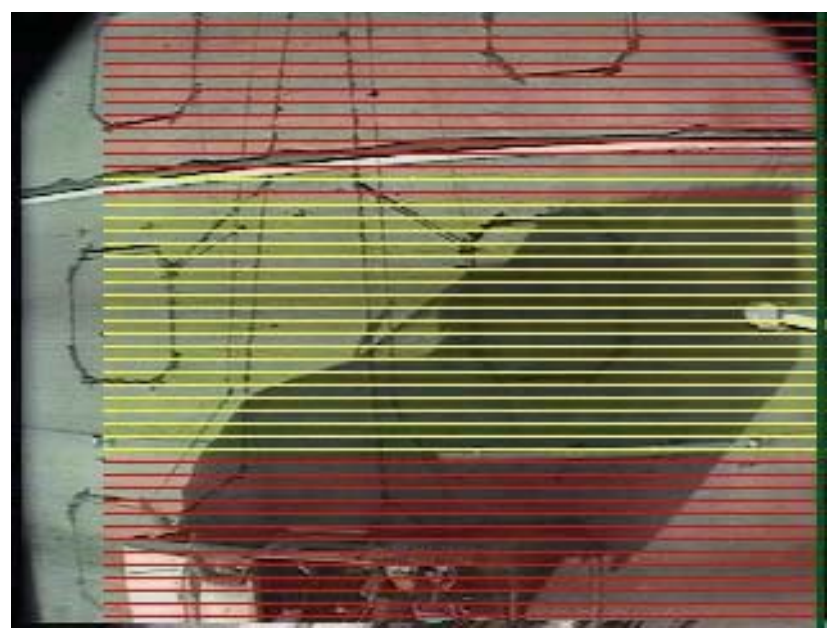

Figure 6. Shadow rejection method illustrated in diagnostic mode. Detection scan lines in lane do not terminate on leading edge of shadow, indicating rejection of shadow flow front.

vehicle, and when available, also measured by duplex loop detectors. When both sources are available, the values are averaged. The time adjustment for vehicle $\mathrm{j}$ reported by detector $i$ is:

$$
t_{i, j}=\frac{x_{i}}{v_{i, j}}
$$

where:

$t_{i, j}$ is the pre-signal delay for the detection (sec.)

$x_{i}$ is the offset of the detection zone from the baseline (m.)

$v_{i, j}=$ the velocity of the vehicle $(\mathrm{m} / \mathrm{s})$

Raw detection times are corrected by subtraction of the pre-signal delay to generate the compensated time of detection. A false detection may not have a corresponding speed measurement if it is not detected by either V2DVS or the duplex loop detector. In this case, the speed of the nearest proximate vehicle in that lane is used.

A reported detection is considered valid if it occurs within a userdefined admissible time/distance aperture, centered about the average compensated time of detection from all detectors, or a designated subset thereof referred to as trusted detectors. The failure of a detector to report a vehicle within the aperture is considered a potential failure-to-detect error, unless a proximate detection is later associated with that grouping during manual verification. Detections occurring outside of an aperture, or multiple detections inside the same non-overlapping aperture, are considered potential false detections. Proximity to the center time is used to discriminate cases of nearly equal admissibility when apertures for closely following vehicles overlap. The aperture for a particular vehicle is illustrated in Figure 7 by lines overlaid on the manual verification window provided by the V2DVS client application. This window graphically depicts on a common time line the results from all detectors for a given lane. Multiple windows can be activated to show multiple lanes concurrently. The client application communicates with the server over a local or Internet connection during the process of manual resolution of ambiguous detections. 
Table 1. Manually verified detection and speed measurement performance of V2DVS for range of lighting conditions.

\begin{tabular}{|l|l|l|l|l|l|}
\hline Illumination condition & $\begin{array}{l}\text { Sample size } \\
\text { (actual vehicles) }\end{array}$ & Correctly detected & Failed to detect & False detected & $\begin{array}{l}\text { Velocity } \\
\text { measurement }\end{array}$ \\
\hline Overhead sun & 300 & $299(99.7 \%)$ & $1(0.3 \%)$ & $0(0 \%)$ & Excellent \\
\hline Diffuse moving shadows & 300 & $298(99.3 \%)$ & $2(0.7 \%)$ & $0(0 \%)$ & Excellent \\
\hline $\begin{array}{l}\text { Crisp moving shadows } \\
\text { from adjacent lane }\end{array}$ & 300 & $295(98.3 \%)$ & $5(1.7 \%)$ & $0(0 \%)$ & Excellent \\
\hline $\begin{array}{l}\text { Fixed (bridge) shadow in } \\
\text { detection zone }\end{array}$ & 200 & $197(98.5 \%)$ & $3(1.5 \%)$ & $0(0 \%)$ & Acceptable \\
\hline $\begin{array}{l}\text { Dappled shadows from } \\
\text { adjacent lane }\end{array}$ & 200 & $197(98.5 \%)$ & $3(1.5 \%)$ & $3(1.5 \%)$ & Acceptable \\
\hline \begin{tabular}{l} 
Low light $(<30 / 255)$ \\
\hline
\end{tabular} & 300 & $298(99.3 \%)$ & $2(0.7 \%)$ & $1(0.3 \%)$ & Acceptable
\end{tabular}

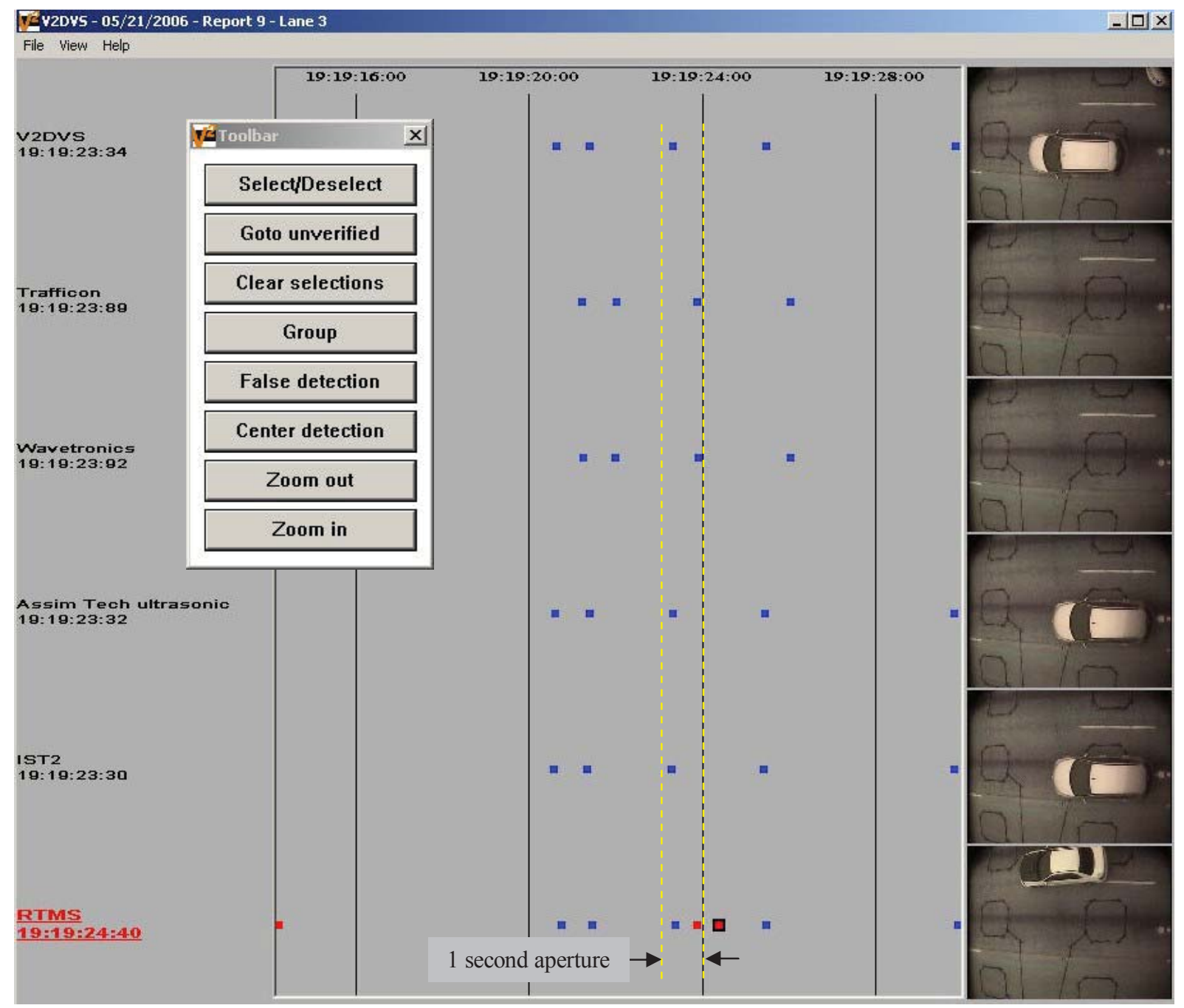

Figure 7. Manual verification window, showing the position-corrected time line for each detector. The correlation aperture for a selected vehicle is overlaid. Automatically correlated detections are blue dots. For the sixth detector, the automatic designation of two false detections is shown by red dots. For the second and third detectors, the detection position was outside camera view. 


\begin{tabular}{|c|c|c|c|c|c|c|c|}
\hline \multicolumn{8}{|l|}{ FIYZDYS - Statistics } \\
\hline & & Lane 1 & Lane 2 & Lane 3 & Lane 4 & Lane 5 & Lane 6 \\
\hline \multirow{3}{*}{ V2Dvs } & Correct & 164 & $\mathbf{0}$ & 239 & 217 & 189 & $\mathbf{0}$ \\
\hline & Fail & 1 & 0 & 1 & 1 & 1 & 0 \\
\hline & False & $\mathbf{0}$ & $\mathbf{0}$ & $\mathbf{0}$ & $\mathbf{0}$ & $\mathbf{0}$ & $\mathbf{0}$ \\
\hline \multirow{3}{*}{ Trafficon } & Correct & 164 & $\mathbf{0}$ & 239 & 209 & 183 & $\mathbf{0}$ \\
\hline & Fail & 1 & $\mathbf{0}$ & 1 & 9 & 7 & $\mathbf{0}$ \\
\hline & False & 0 & $\mathbf{0}$ & 8 & 0 & $\mathbf{0}$ & $\mathbf{0}$ \\
\hline \multirow{3}{*}{ Wavetronics } & Correct & 156 & $\mathbf{0}$ & 230 & 206 & 182 & 0 \\
\hline & Fail & 9 & 0 & 10 & 12 & 8 & 0 \\
\hline & False & 0 & $\mathbf{0}$ & 11 & 0 & 0 & $\mathbf{0}$ \\
\hline \multirow{3}{*}{ Assim Tech ultrasonic } & Correct & 159 & $\mathbf{0}$ & 235 & 0 & 184 & $\mathbf{0}$ \\
\hline & Fail & 6 & $\mathbf{0}$ & 5 & 218 & 6 & 0 \\
\hline & False & $\mathbf{0}$ & $\mathbf{0}$ & 22 & $\mathbf{0}$ & 0 & $\mathbf{0}$ \\
\hline \multirow{3}{*}{ IST2 } & Correct & 163 & 0 & 240 & 213 & 184 & $\mathbf{0}$ \\
\hline & Fail & 2 & 0 & 0 & 5 & 6 & 0 \\
\hline & False & 0 & 0 & 3 & 0 & 0 & 0 \\
\hline \multirow{3}{*}{ RTMS } & Correct & 68 & 0 & 228 & 180 & 107 & 0 \\
\hline & Fail & 97 & 0 & 12 & 38 & 83 & 0 \\
\hline & False & 0 & 0 & 35 & 0 & 0 & 0 \\
\hline
\end{tabular}

Figure 8. Statistics window showing sample of results from preliminary system testing in four lanes. Numbers shown are not necessarily representative of actual performance of listed detectors.

The manual verification display shown in Figure 7 shows the results of successful automatic correlation as blue dots, false detections in red. Uncertain detections (not shown) are yellow. Mouse-click selection of any dot brings up the corresponding image acquired at the time of that detection, which makes it clear if the detection was correct, or in what way incorrect. The status of any detection (colored dot), including those that may have been incorrectly grouped by the automated process, can be changed by clicking on the toolbar buttons shown in the inset window.

Once manual verification has been completed, either for an individual lane or across all lanes, the dataset is marked by the operator as closed, and V2DVS generates automatic test results for all detectors under test. The "Statistics" window is shown in Figure 8 for a preliminary test run conducted prior to the optimization of the detectors under test.

\section{OBSERVATIONS AND CONCLUSIONS}

Automated data reduction greatly reduces the workload associated with ground truth generation, since it requires human verification only for detections that cannot be automatically correlated. In preliminary testing with 1600 vehicles (9600 detections) under various conditions, approximately $97 \%$ of vehicle detections were properly classified as correct, false, or failure to detect by the automated process. Errors were most commonly related to ambiguous vehicle lane position or an excess of false detections by one or more of the detectors under test. Accuracy is dependent upon the size of the admissible time/distance aperture, with more conservative settings tending to reject valid detections, while less conservative settings admitting incorrect matches which sometimes cause misalignment errors that propagate to other proximate vehicles in the ground truth data set. Further details may be obtained from [7].

\section{ACKNOWLEDGEMENTS AND DISCLAIMER}

This work was supported by the California Department of Transportation via the California PATH (Partners for Advanced Transit and Highways) program. Statements and results reported herein are the responsibility of the authors exclusively. This report does not constitute a standard, specification or regulation.

\section{REFERENCES}

[1] Loragen Corporation, Instrumentation and Evaluation of the Caltrans Automated Warning System, Final report to the California Dept of Transportation, Contract No. 51A0050, OTS Grant No. RS0034, Doc No. F-D10-2004/5, July 22, 2005. [2] James H. Kell, Iris J. Fullerton, Milton K. Mills, Traffic Detector Handbook, U.S. Department of Transportation Federal Highway Administration, Office of Research and Development, FHWA-IP-90-002, July 1990.

[3] US Dept. of Transportation, Field Test of Monitoring of Urban Vehicle Operations Using Non-Intrusive Technologies, FHWAPL-97-018, 1997.

[4] DuBois, Paul, MySQL, $2^{\text {nd }}$ ed.. Pearson Education, ISBN 07357-1212-3, Jan 2003.

[5] Cho, Jeong-Hoon,Kwon, Tae-Gyun; Jang, Dae-Geun; Hwang, Chan-Sik Advances in Artificial Intelligence, Proc. 18th Australian Joint Conf. on Artificial Intelligence, 2005, p 746-755.

[6] Xu, Haixiang; Zhu, Guangxi; Peng, Fuyuan; Ge, Wei; Xie, Lei; Tao, Pingan Proc. SPIE, v 6044, MIPPR 2005: Image Analysis Techniques, 2005, p. 60441T.

[7] MacCarley, C.A., Video Vehicle Detector Verification System (V2DVS) Operators Manual, Calif. Dept. of Transportation Task Order 5327, Cal Poly Corp. Project No. 48020, May 18, 2006. 